https://doi.org/10.23913/rics.v8i16.81

Artículos Científicos

\title{
Hipoterapia: alternativa para el abordaje terapéutico de las discapacidades neuromotoras
}

Hypotherapy: alternative for the therapeutic approach of neuromotor

disabilities

Hipoterapia: alternativa para a abordagem terapêutica das deficiências neuromotoras

Salvador Ernesto Sarmiento Vega

Universidad Veracruzana, México ssarmiento@uv.mx https://orcid.org/0000-0002-7382-0312

María Esther Barradas Alarcón

Universidad Veracruzana, México ebarradas@uv.mx https://orcid.org/0000-0002-0208-9083

Martha Lilia León Noris Universidad Veracruzana, México mleon@uv.mx https://orcid.org/0000-0002-9833-9859

Javier López González Universidad Veracruzana, México jalopez@uv.mx https://orcid.org/0000-0003-2704-7944

Mayra Helen Posadas Tello

Universidad Veracruzana, México mposadas@uv.mx https://orcid.org/0000-0003-2174-8205 


\section{Resumen}

El objetivo de este estudio fue analizar la interrelación entre los elementos participantes en la tríada terapéutica (cliente o paciente, equino y facilitador) durante el proceso terapéutico de un paciente masculino de 27 años con diagnóstico de lesión medular C6-7. Para ello, se empleó la hipoterapia como una propuesta alternativa y complementaria para el tratamiento de personas con o sin discapacidad; esta es una actividad que promueve el potencial humano de desarrollo utilizando al caballo y a su entorno como la base de la terapia. La investigación fue planteada desde la perspectiva de la investigación cualitativa. Por sus características se considera como un trabajo de carácter exploratorio. El método empleado se ajusta a los postulados del modelo de la teoría fundamentada. El tipo de estudio fue el análisis de caso clínico. Para la recolección de datos se empleó la técnica documental biográfica. La información fue procesada mediante la técnica de análisis de contenido, con apoyo en las perspectivas teóricas de la neurofisiología aplicada al campo de la hipoterapia, la comunicación interespecies (humano-equino) y el enfoque centrado en la persona. Las observaciones obtenidas del análisis del discurso muestran claramente el cumplimiento de los principios señalados para cada uno de los modelos teóricos empleados. De hecho, los resultados obtenidos son similares a los reportados por otros autores en cuanto a la utilidad del caballo como coterapeuta en virtud de sus características y reacciones, las cuales poseen una importante similitud con las condiciones terapéuticas descritas en el modelo centrado en la persona para facilitar el proceso de cambio, es decir, la aceptación incondicional, la congruencia y la empatía.

Palabras clave: equinoterapia, equus diálogo, discapacidad. 


\section{Abstract}

The objective of this study was to analyze the interrelation between the elements participating in the therapeutic triad (client or patient, equine and facilitator) during the therapeutic process of a 27-year-old male patient diagnosed with spinal cord injury C6-7. For this, hippotherapy was used as an alternative and complementary proposal for the treatment of people with or without disabilities; This is an activity that promotes human development potential using the horse and its environment as the basis of therapy. The research was raised from the perspective of qualitative research. Due to its characteristics, it is considered as an exploratory work. The method used conforms to the postulates of the grounded theory model. The type of study was the clinical case analysis. The biographical documentary technique was used to collect data. The information was processed using the content analysis technique, with support in the theoretical perspectives of neurophysiology applied to the field of hypotherapy, interspecies communication (human-equine) and the person-centered approach. The observations obtained from the discourse analysis clearly show compliance with the principles indicated for each of the theoretical models used. In fact, the results obtained are similar to those reported by other authors regarding the usefulness of the horse as a co-therapist by virtue of its characteristics and reactions, which have an important similarity with the therapeutic conditions described in the person-centered model for facilitate the process of change, that is, unconditional acceptance, congruence and empathy.

Keywords: equine therapy, equus dialogue, disability.

\section{Resumo}

O objetivo deste estudo foi analisar a inter-relação entre os elementos participantes da tríade terapêutica (cliente ou paciente, equino e facilitador) durante o processo terapêutico de um paciente de 27 anos com diagnóstico de lesão medular C6-7. Para isso, a hipoterapia foi utilizada como uma proposta alternativa e complementar para o tratamento de pessoas com ou sem deficiência; Esta é uma atividade que promove o potencial de desenvolvimento humano usando o cavalo e seu ambiente como base da terapia. A pesquisa foi levantada na perspectiva da pesquisa qualitativa. Por suas características, é considerado um trabalho exploratório. O método utilizado está em conformidade com os postulados do modelo da 
teoria fundamentada. O tipo de estudo foi a análise de caso clínico. A técnica documental biográfica foi usada para coletar dados. As informações foram processadas pela técnica de análise de conteúdo, com suporte nas perspectivas teóricas da neurofisiologia aplicada ao campo da hipoterapia, comunicação interespécies (equino-humano) e abordagem centrada na pessoa. As observações obtidas na análise do discurso mostram claramente o cumprimento dos princípios indicados para cada um dos modelos teóricos utilizados. De fato, os resultados obtidos são semelhantes aos relatados por outros autores em relação à utilidade do cavalo como co-terapeuta em virtude de suas características e reações, que possuem uma importante semelhança com as condições terapêuticas descritas no modelo centrado na pessoa para facilitar o processo de mudança, isto é, aceitação incondicional, congruência e empatia.

Palavras-chave: equoterapia, diálogo equus, incapacidade.

Fecha Recepción: Enero 2019

Fecha Aceptación: Junio 2019

\section{Introducción}

Para referirnos a la utilización del caballo como un recurso de apoyo a la salud es necesario mencionar, en primer término, que se trata de una relación que tiene sus raíces en una práctica milenaria (Burbridge, 2004) que ha sido registrada en crónicas y leyendas que dan testimonio del invaluable aporte que han tenido los equinos en cada uno de los escaños evolutivos del ser humano. De hecho, y a pesar del actual dominio de la ciencia y la tecnología en los ámbitos de la vida cotidiana, el caballo sigue siendo un símbolo universal en torno al cual se sigue tejiendo el enramado histórico trasmitido a través de crónicas que subliman las cualidades de estos animales no solo en términos de belleza, arrojo, gracia y fuerza, sino también de nobleza y fidelidad.

Estas precisiones nos permiten legitimar, en cierto modo, la adopción de una práctica vigente en el colectivo histórico, aunque "redescubierta" en años recientes, la cual exalta los indudables beneficios terapéuticos que se derivan de las terapias asistidas con equinos. En tal sentido, y sin que esto constituya una definición, se puede asegurar — según lo señalado por autores como Gross (2006), Ernst y De la Fuente (2007), Castillo (2011) y Bender (2012)

\section{(c) $\rightarrow$ Ho}

Esta obra está bajo licencia internacional Creative commons Reconocimiento-Nocomercial- 
que las terapias ecuestres son aquellas actividades que utilizan al caballo como un asistente mediante el cual se puede promover el desarrollo cognitivo, físico, emocional, social y ocupacional de personas que tengan o no alguna discapacidad.

El concepto terapias ecuestres es un enunciado general utilizado para caracterizar a una disciplina naciente que incluye al menos a dos tipos de práctica con aplicaciones y estrategias diferentes: la equinoterapia y la psicoterapia asistida con equinos. Más allá de los objetivos y métodos aplicados, ambos métodos tienen en común su praxis, la cual se desarrolla en un entorno natural que proporciona un campo perceptivo más amplio y diverso que el experimentado en las prácticas tradicionales, lo que contribuye a la consecución de los objetivos propuestos (Pérez, Rodríguez y Rodríguez, 2008).

Apuntado lo anterior, valga precisar que el énfasis en este trabajo ha recaído en el proceso de la equinoterapia, para lo cual se ha tomado como punto de análisis la interrelación de los elementos participantes (cliente, equino y facilitador).

\section{Equinoterapia}

Por sus particularidades, la equinoterapia se puede caracterizar como una forma de terapia alternativa y complementaria que utiliza al caballo como un recurso natural al que se puede recurrir para brindar asistencia a personas con capacidades diferentes. De este modo se intenta incidir en el mejoramiento de la calidad de vida de las personas para promover su integración a la sociedad (Bender, 2012; Ernst y De la Fuente, 2007; Gross, 2006).

En el Congreso Internacional de la Monta Terapéutica —celebrado en 1998 en Toronto, Canadá- se ha propuesto la división de la equinoterapia en tres áreas o campos de trabajo (Castillo, 2011):

a. Hipoterapia.

b. Monta terapéutica.

c. Equitación como deporte para discapacitados.

En relación con su práctica, autores como Caudet (2002), Gross (2006), Pérez, Rodríguez y Rodríguez (2008), Castillo (2011) y Bender (2012), entre otros, señalan que se suele preferir al caballo en las prácticas terapéuticas, en lugar de otras especies, debido a tres

\section{(C) $\rightarrow$ FO}

Esta obra está bajo licencia internacional Creative commons Reconocimiento-NoComercial- 
particularidades inherentes a su naturaleza, las cuales actúan de manera concurrente sobre el jinete, independientemente de la condición de discapacidad que presente:

a. La trasmisión del calor corporal.

b. La trasmisión de impulsos rítmicos.

c. La trasmisión de un patrón de locomoción equivalente al patrón fisiológico de la marcha humana.

La conjunción de estas acciones durante la monta produce efectos terapéuticos a nivel neuromotor, sensomotor, psicomotor, sociomotor y funcional.

Ahora bien, con la finalidad de dejar clara la posición desde la cual se ha enfocado el presente trabajo, es necesario señalar que el concepto discapacidad se vincula solo con la disminución de una destreza en algún área específica, con lo cual se intenta erradicar el matiz prejuicioso de minusvalía que se halla implícito en otras acepciones.

Según el Enfoque Centrado en la Persona, Rogers (1993) considera que el cuerpo de un individuo es solo un soporte donde se encuentran una infinidad de capacidades potenciales que, al ser liberadas y desarrolladas, inscriben al ser humano en una dinámica de cambio constante; a esto Rogers denomina proceso de convertirse en persona. A partir de esta premisa, se puede asegurar que una persona puede presentar algún tipo de inhabilidad, pero no es su discapacidad, por lo que tiene el mismo potencial para desarrollar cualquier destreza con la única limitante que su propia condición le imponga.

\section{Metodología}

La presente investigación fue de naturaleza cualitativa porque - a diferencia del enfoque cuantitativo - permite empelar técnicas para recoger y analizar la experiencia y el discurso de los sujetos, los cuales se hallan inmersos en su contexto natural (Hernández, Fernández, Baptista, 2000). El objetivo del estudio fue analizar la interrelación de los

elementos participantes (cliente, equino y facilitador) durante el proceso terapéutico de un paciente masculino de 27 años con diagnóstico de lesión medular C6-7.

El método empleado fue la teoría fundada o fundamentada, la cual básicamente es un diseño metodológico que sirve para explicar un segmento de realidad en su ámbito original; es decir, se pueden analizar los elementos del discurso y el significado de la experiencia (c) 9 SP 
mediante la interrelación de los principios teóricos que subyacen en la expresión subjetiva del narrador (Bautista, 2011). Esto sirve para relacionar el conocimiento previo con las características específicas del fenómeno.

El tipo de estudio fue el análisis de caso clínico debido a que la propia naturaleza del problema lo ubica en el ámbito de la salud (Artiles, Artiles y Rodríguez, 2016). Para la recolección de datos se empleó la técnica documental biográfica (Bautista, 2011), mientras que para el procesamiento de la información se recurrió a la técnica de análisis de contenido, el cual se sustentó en las perspectivas teóricas de la neurofisiología aplicada al campo de la hipoterapia, la comunicación interespecies (humano-equino) y el enfoque centrado en la persona. Para facilitar el análisis de los datos se adaptó el modelo utilizado por Barreiro (2015), el cual consta básicamente de dos momentos: el primero corresponde al tiempo en que el investigador suspende la lectura del texto/discurso para evaluar (por fragmentos) la vivencia experiencial desde la perspectiva del narrador; el segundo momento consiste en relacionar dicha vivencia con los posicionamientos teóricos desde los cuales se intenta explicar o comprender el fenómeno.

\section{Presentación de caso de estudio}

Características del paciente: Masculino de 27 años con diagnóstico de lesión medular C6-7.

\section{Características de la terapia}

Por las características del paciente se trabajó con la modalidad de la hipoterapia pasiva o básica (Bender, 2012; Gross, 2006). La duración de las sesiones fue de una hora aproximadamente, de la cual entre 25 y 30 minutos (efectivos) se consideraron para el trabajo de monta, así como pausas para descansar y realizar ejercicios respiratorios; el resto fue utilizado para el establecimiento de los contactos (al inicio y al final de la sesión) en los cuales el paciente podía acariciar al caballo y darle algunas zanahorias como premio.

Al finalizar la sesión el paciente tenía la oportunidad de permanecer un momento de pie sujetándose del lomo del caballo, para lo cual fue asistido por los ayudantes de piso antes de volver a su silla. Los ejercicios y los cuidados de sujeción del paciente responden a los 
criterios señalados por Gross (2006) y Bender (2012). Se trabajaron con ejercicios en posición de montado (al frente y hacia atrás) y atravesado sobre el lomo del caballo en posición decúbito prona.

\section{Duración de tratamiento}

El tratamiento tuvo una duración aproximada de tres meses, tiempo en el cual se tuvieron 12 sesiones (una vez por semana). El tratamiento fue interrumpido por necesidades personales del paciente.

\section{Perspectivas teóricas para el análisis de datos}

El análisis de los datos se realizó a través de tres filtros teóricos:

a. Los supuestos o principios inherentes a la equinoterapia que nos permiten explicar el proceso de la rehabilitación y los efectos terapéuticos esperados para cada una de las áreas de funcionamiento involucradas.

b. Los supuestos o constructos contenidos en el enfoque centrado en la persona (ECP) utilizados de manera análoga en el análisis del proceso interaccional de la triada terapéutica (cliente, caballo y terapeuta); al respecto, cabe mencionar que en el presente documento se han usado indistintamente los términos cliente y paciente, así como los de facilitador y terapeuta.

c. Se propone la formulación del equus-diálogo ${ }^{1}$ como un concepto innovador basado en el aprendizaje de la comunicación interespecies. Este ofrece la posibilidad de interpretar, de manera recíproca, los códigos de comunicación de los equinos con la finalidad de comprender el establecimiento de vínculos afectivos en los cuales se pueden reconocer no solo la expresión de las emociones básicas para la supervivencia, sino también los sentimientos involucrados en el encuentro o condición del contacto humano-equino; en todo caso, la dirección del encuentro (aproximación-alejamiento) depende en parte tanto de las disposiciones caracterológicas de la persona en cuestión

\footnotetext{
${ }^{1}$ El equus-diálogo se presenta como un concepto revelador del proceso dialógico que transcurre desde el momento que inicia la lectura corporal recíproca entre el equino y su interlocutor humano hasta el establecimiento de un vínculo generado por un mecanismo de identificación proyectiva.
} 


\section{Revista Iberoamericana de las Ciencias de la Salud}

como de las disposiciones caracterológicas o brío del equino (Alonso, 2017, comunicación personal).

\section{Análisis y correspondencia teórica del texto}

Mi nombre es... Llevo 11 años en silla de ruedas como consecuencia de una fractura a nivel cervical. A lo largo de estos años he probado con distintos tipos de terapias y cada una ha aportado algún beneficio en mi recuperación; sin embargo, la equinoterapia me ha brindado más "cosas" que ninguna otra []$^{1}$. Digo "cosas" puesto que es complicado de explicar, sin embargo, intentaré explicar lo que me hace sentir []$^{2}$.

\section{Adscripción teórica}

[ ] ${ }^{1}$ En el contexto de esta frase se puede reconocer la condición o estado de contacto señalada por Rogers y Gelind (citados por Barceló, 2012); igualmente, la interpretación de esa línea representa un cambio positivo en la percepción del yo mismo así como una clara atribución a su experiencia del proceso.

[ ] $]^{2}$ En esta línea se aprecia la condición de restablecimiento de la congruencia entre el yo y la experiencia actual (Rogers, 1998), que tácitamente revela una situación de fortaleza ante las experiencias amenazantes. Simplificando, se puede decir que a menor sentimiento de amenaza mayor consideración positiva hacia los demás. En esta condición la adaptación psicológica se ve aumentada, y la valoración organísmica actúa cada vez más como la base de regulación de la conducta.

Desde el primer momento que llegué al hípico ya mi percepción había recibido la calma y la tranquilidad de los árboles y la naturaleza, la frescura y el silencio del lugar []$^{3}$; estos elementos me desestresaron y me relajaron casi de golpe. Después de platicar con el maestro de cómo me subirían al caballo (lo cual nunca es fácil) procedimos a realizarlo []$^{4}$.

\section{(c) $\rightarrow \& \Theta$}




\section{Revista Iberoamericana de las Ciencias de la Salud}

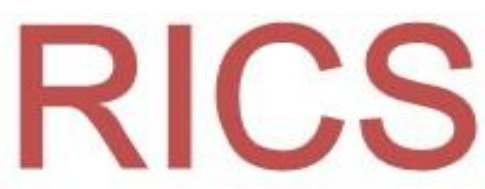

ISSN: $2395-8057$

[ ] $]^{3}$ Este segmento indica, de acuerdo con lo señalado por Pérez y cols. (2008), que el efecto positivo del contexto natural en el que transcurren las sesiones favorece la reducción de las condiciones de estrés. Además, el campo perceptivo constituido por estímulos novedosos amplían las posibilidades de la experiencia del contacto.

[ ] $]^{4}$ El contexto general de la frase y el énfasis expuesto en esta última afirmación confirma la presencia de la condición de apertura a la experiencia formulada por Roger (1998), quien señala que cuanto más segura se sienta una persona más abierta estará a la experiencia.

Ese momento fue cumbre en mi vida, porque tenía frente a mí a un animal enorme y el hecho de darle azúcar, de sentir su lengua tocando mis manos, portándose de una manera dócil y despreocupada me hizo sentir seguro, animado y contento [ ] $]^{5}$.

[ $]^{5}$ En el contexto general de la frase se observa la pertinencia del equus-diálogo en la formación de impresiones cognitivo/afectivas autorreguladas, las cuales, por una parte, revelan la presencia de las condiciones de aceptación incondicional y de empatía propuestas por Rogers (1988). Esto, además, permite reforzar la posibilidad del diálogo interespecies (Roberts, 2006).

$\mathrm{Al}$ subirme en Coronela por primera vez, tuve un mar de emociones, todas positivas, desde la sensación de logro [ $]^{6}$ debido a que jamás me imaginé subirme a un caballo, sentí una especie de liberación de la silla de ruedas, ya que el movimiento, la altura, la perspectiva, todo era nuevo y emocionante []$^{7}$.

[ ] ${ }^{6}$ En estas líneas se refleja el constructo relativo a la actualización del yo. Según Rogers (1988), la congruencia entre el yo y la experiencia puede ser reestablecida a través de la tendencia a la actualización del yo. En ese sentido, se está presenciando un momento de revaloración en torno a la percepción del yo mismo; es decir, de su autoconcepto, de su autoimagen y de su autoestima.

\section{(c) $\rightarrow \& \Theta$}




\section{Revista Iberoamericana de las Ciencias de la Salud}

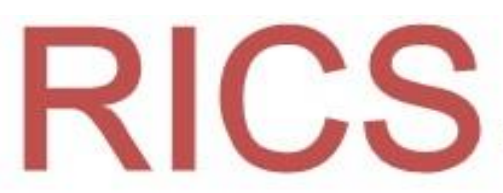

ISSN: $2395-8057$

[ ] $]^{7}$ Este enunciado permite apreciar, de acuerdo con lo señalado por Gross (2006), el efecto supuesto en el primer principio de la equinoterapia en relación con las sensaciones de seguridad, aceptación y protección.

Ese día cerré mis ojos e imaginé que caminaba, ya que sentía como se movía mi cadera y mis piernas como hacía mucho no sentía []$^{8}$.

[ $]^{8}$ Este segmento remite a lo señalado por Strauss (citado por Gross, 2006) no solo sobre la grabación del patrón de marcha, sino también de la sensación de movimiento generado por la marcha del equino (principio de locomoción; efecto psicomotor). Asimismo, se vincula con el desarrollo del tono muscular, la flexibilidad de los ligamentos pélvicos y a la estabilización del balanceo dinámico del tronco y la cabeza.

A lo largo de 12 sesiones he desarrollado un vínculo con Coronela, ella es capaz de percibir mi nivel de agotamiento y mi agitación durante los ejercicios [ ] ${ }^{9}$.

[ ] $]^{9}$ Lo expresado en estas líneas tiene un gran valor porque demuestra tanto la condición de contacto descrita por Barceló (2012) como el estado o condición de empatía referido por Rogers (1998) en torno a la correcta percepción del marco de referencia del otro.

Rebufa cuando me encuentro relajado y respirando profundamente []$^{10}$.

[ $]^{10}$ En este señalamiento se puede apreciar la idea (por parte del paciente) de una respuesta de reconocimiento (del equino) de la condición emocional y el estado físico del cliente/paciente, la cual es generada por la elaboración de un proceso de identificación proyectiva $^{2}$.

\footnotetext{
${ }^{2}$ En el discurso conservador de las psicoterapias, la identificación proyectiva está referida al proceso transferencial entre el paciente y el terapeuta; sin embargo, para efectos de este trabajo, se ha empelado de manera analógica para describir el vínculo afectivo (positivo o negativo) que se genera durante el proceso de cambio en la tríada terapéutica cliente, caballo y facilitador.
}

\section{(c) 989}




\section{Revista Iberoamericana de las Ciencias de la Salud}

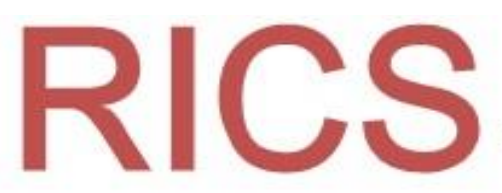

También he recuperado sensibilidad en la espalda baja, piernas, glúteos e incluso a nivel de esfínteres [ $]^{11}$.

[ ] ${ }^{11}$ En esta frase se nota el efecto supuesto en el segundo principio de la equinoterapia (trasmisión de impulsos rítmicos), en tanto que los impulsos producidos por el movimiento del caballo a la pelvis tocan también las piernas del jinete y provocan un relajamiento de los músculos aductores y los ligamentos pélvicos. De hecho, estimulan la erección de la columna vertebral y la funcionalidad de los esfínteres (Gross, 2006).

Actualmente tengo una mejor condición física, logro estar más erguido al estar sentado, mis brazos también han aumentado su masa muscular [ $]^{12}$.

[ ] ${ }^{12}$ El contenido de este segmento remite al constructo enunciado por Rogers (1998), es decir, la tendencia organísmica de actualización en la que se presupone la tendencia innata del organismo para desarrollar sus potencialidades, bien sea para conservarlo o para mejorarlo.

Hasta aquí, lo escrito solo son algunas de las cosas que he obtenido, sin embargo, el mayor reconocimiento que le doy a la equinoterapia es el que a diferencia de otras no trabaja solo con mi cuerpo como si solo fuese un objeto que necesita moverse [ $]^{13}$, la equinoterapia me permite recuperar sensibilidad corporal y experimentar emociones diferentes: felicidad, logros, orgullo, cariño, bienestar, entre otras emociones []$^{14}$ que, sinceramente, son necesarias para seguir adelante no solo con la terapia, sino también con la vida []$^{15}$.

[ $]^{13}$ En este fragmento se observa la condición de un yo actualizado (Rogers, 1998) porque revela un reconocimiento integral por parte del paciente de su propia percepción de discapacidad en la que la parte motora es solo una de su ser como persona.

[]$^{14}$ En esta idea se aprecia la condición de actualización del yo mencionada, cuya actuación, para este caso, involucra tanto al campo organísmico como al psicológico de la percepción del yo mismo. Asimismo, se evidencia - al menos hipotéticamente - el resultado 


\section{Revista Iberoamericana de las Ciencias de la Salud}

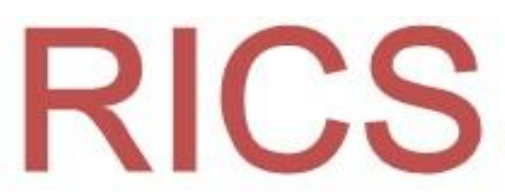

de la conjugación de los diferentes principios y los efectos terapéuticos consecuentes en los que se sustenta la equinoterapia (Gross, 2006).

[ ] ${ }^{15}$ En el colofón del documento se advierte lo reportado por Längle (2008), es decir, la emoción por el descubrimiento de un sentido ${ }^{3}$ qué tácitamente expresa una elección responsable acerca de cómo enfrentar la cotidianidad de la vida.

\section{Conclusiones}

\section{De la estrategia metodológica}

El encuadre metodológico usado permitió analizar de manera puntual el discurso del cliente/paciente desde los tres ámbitos implícitos en el proceso de la equinoterapia, esto es, la fisiología, la comunicación interespecies y la postura humanista de la psicología.

Desde luego, afirmar la correspondencia puntual entre el discurso y las conceptualizaciones teóricas de nuestros referentes analíticos resulta una tarea casi imposible, puesto que la formación de juicios respecto al sentido de las percepciones afectivas de los eventos está condicionada por elementos bio-psico-sociales-espirituales (Sarmiento, 2003) implícitos en las elaboraciones cognitivas con que se simboliza la experiencia personal.

No obstante lo anterior, se debe señalar la afinidad de las afirmaciones contenidas en el discurso con los campos temáticos utilizados para el análisis:

Llevo 11 años en silla de ruedas como consecuencia de una fractura a nivel cervical. A lo largo de estos años he probado con distintos tipos de terapias y cada una ha aportado algún beneficio en mi recuperación; sin embargo, la equinoterapia me ha brindado más "cosas" que ninguna otra. Digo "cosas", puesto que es complicado de explicar, sin embargo, intentaré explicar lo que me hace sentir.

\footnotetext{
${ }^{3}$ Se ha adoptado la formulación de Längle (2008) para describir la revelación de un sentido en los términos propuestos por el mismo autor: la actualidad de la búsqueda del sentido radica, primero, en la libertad del ser humano, en su condición de inacabado; segundo, en la valencia diferente de las cosas y, tercero, en el cambio constante de las situaciones.
}

\section{(c) 9 (9)}


El contexto general de la frase refiere de una manera muy precisa al sentido de lo propuesto por Rogers y Gelind (citados por Barceló, 2012) sobre la condición de contacto que de hecho constituye uno de los constructos vertebrales del enfoque - en el que se afirma que el estar de dos personas (una en presencia de la otra) afecta de manera recíproca el campo experiencial de ambas.

En la declaración de Rogers y Gelind este concepto destaca claramente la importancia de las actitudes del terapeuta (por sobre los métodos y las técnicas) como la vía para el cambio y el crecimiento personal. La atribución de excepción respecto al beneficio de la equinoterapia que el paciente declara proporciona la evidencia de un cambio positivo en la percepción del yo mismo. En este sentido, se puede indicar la disminución de la percepción de amenaza como resultado de la capacidad organísmica de autorregulación, que reorienta la condición de vulnerabilidad hacia la adaptación psicológica, situación a la que Rogers (1998) se refiere como restablecimiento de la congruencia entre el yo y la experiencia.

Desde el primer momento que llegué al hípico ya mi percepción había recibido la calma y la tranquilidad de los árboles y la naturaleza, la frescura y el silencio del lugar; estos elementos me desestresaron y me relajaron casi de golpe.

Esta frase deja entrever una gran riqueza debido a la vinculación que tiene con las afirmaciones de los autores referenciados en este análisis. En otras palabras, la impresión de asombro y redescubrimiento del patrón de marcha (por ejemplo) remite, en primera instancia, a lo declarado por Pérez Rodríguez y Rodríguez (2008) sobre el efecto positivo de la equinoterapia. Al respecto, este autor explica que las actividades desarrolladas en un espacio abierto y en un ambiente natural tienen un efecto favorable en la esfera psicológica y emocional del paciente y fomentan la creación de vínculos entre el cliente y su entorno natural y social.

En concordancia con esta idea, Gross, (2006) menciona que el andar rítmico y suave del caballo provoca un efecto de "mecedora", el cual — sumado a la temperatura corporal del caballo - evoca sentimientos de seguridad, amor y protección en el jinete. Además, es clara la condición de contacto mencionada entre el facilitador, el cliente, el equino y su entorno.

\section{(c) $\rightarrow$ Ho}

Esta obra está bajo licencia internacional Creative commons Reconocimiento-Nocomercial- 


\section{Revista Iberoamericana de las Ciencias de la Salud}

Después de platicar con el maestro de cómo me subirían al caballo (lo cual nunca es fácil) procedimos a realizarlo.

Este segmento demuestra ese impacto particular de la condición de contacto ya referida, aunque en este caso la referencia está dirigida hacia cómo la conexión entre el cliente y el facilitador generan sentimientos de confianza. Este vínculo progresa a medida que transcurre el proceso, lo cual, de facto, contribuye a la gestación del cambio terapéutico (Rogers, 1993).

Ese momento fue cumbre en mi vida, porque tenía frente a mí a un animal enorme y el hecho de darle azúcar, de sentir su lengua tocando mis manos, portándose de una manera dócil y despreocupada me hizo sentir seguro, animado y contento.

Este es un momento verdaderamente importante en relación con el concepto equusdiálogo porque refleja de manera tácita la interpretación (por parte del paciente) de los movimientos y las actitudes del caballo en términos de la relación cálida percibida por el jinete, así como la condición de aceptación incondicional y empatía (Rogers, 1998). En este proceso se evidencia una actitud exenta de juicios y una percepción de comprensión respecto a las limitaciones impuestas por su discapacidad.

Igualmente, se puede notar que el proceso de identificación proyectiva condiciona los sentimientos experimentados por el jinete. Sobre este asunto, Netscher y Samaniego (2012) señalan — en torno a las actitudes de congruencia, honestidad y empatía - una disposición especial que sirve para reflejar los sentimientos de los pacientes. Por ende, se puede concluir que el contacto emocional entre el binomio jinete-equino, como ya se ha mencionado, es una muestra el diálogo interespecies (Roberts, 2006; Gutiérrez, Granados y Piar 2007). En pocas palabras, más que una simple probabilidad, este es un hecho constatable.

\section{(c) 9 क्ष}




\section{Revista Iberoamericana de las Ciencias de la Salud}

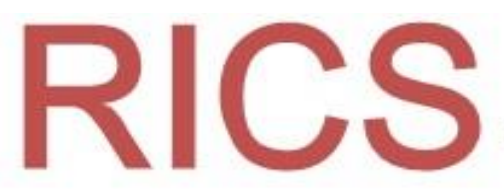

ISSN: $2395-8057$

Al subirme en Coronela por primera vez, tuve un mar de emociones, todas positivas, desde la sensación de logro, debido a que jamás me imaginé subirme a un caballo, sentí una especie de liberación de la silla de ruedas, ya que el movimiento, la altura, la perspectiva, todo era nuevo y emocionante, ese día cerré mis ojos e imaginé que caminaba, ya que sentía como se movía mi cadera y mis piernas como hacía mucho no sentía.

Este segmento es igualmente importante por el tratamiento emocional que da a la experiencia; a través del contenido de lo expuesto por el cliente, se evidencia una clara correspondencia con la condición de actualización del yo (Rogers, 1988), la cual consiste esencialmente en un incremento de la congruencia entre el yo y la experiencia. Esto genera la revaloración del yo mismo respecto a la percepción del autoconcepto, la autoimagen y, desde luego, la autoestima. En este mismo texto se destaca, sobre las sensaciones del paciente de "estar caminando", lo observado por Straus (citado por Gross, 2006) en torno a la grabación neuronal del patrón de marcha, la sensación de movimiento generada por el andar del equino (efecto psicomotor del principio de locomoción), el desarrollo del tono muscular, la flexibilidad de los ligamentos pélvicos y la estabilización del balanceo en la dinámica tronco-cabeza.

A lo largo de 12 sesiones he desarrollado un vínculo con Coronela, ella es capaz de percibir mi nivel de agotamiento y mi agitación durante los ejercicios, rebufa cuando me encuentro relajado y respirando profundamente.

De acuerdo con lo referido por Barceló (2012), la mención al desarrollo del vínculo con el caballo confirma el hecho de que el contacto terapéutico únicamente puede darse cuando ambos participantes están dispuestos a comprometerse en una relación de ayuda terapéutica.

Por otra parte — de acuerdo con lo expresado por grandes expertos en el manejo de los caballos, como Nestcher y Samaniego (2012), Roberts (2006) y Gutiérrez y col (2007) —, se reconoce el desarrollo del sentimiento de empatía (identificación proyectiva positiva) en la declaración del paciente de la capacidad del equino para percibir las expresiones físicas 


\section{Revista Iberoamericana de las Ciencias de la Salud}

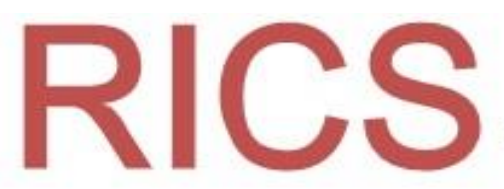

ISSN: $2395-8057$

de agotamiento, excitación o relajación mediante la emisión de sonidos con los cuales el caballo espejea los movimientos corporales del jinete.

También he recuperado sensibilidad en la espalda baja, piernas, glúteos e incluso a nivel de esfínteres. Actualmente tengo una mejor condición física, logro estar más erguido al estar sentado, mis brazos también han aumentado su masa muscular.

Esta afirmación sobre el mejoramiento de la condición física confirma lo expresado por Gross (2006) en relación con que la trasmisión de los impulsos rítmicos del caballo hacia la pelvis, pues al tocar las piernas del jinete generan un efecto de relajación de los músculos aductores y los ligamentos pélvicos y estimula la erección de la columna vertebral y la funcionalidad de los esfínteres. En esa misma línea, Rogers (1998) al plantear su constructo tendencia organísmica de actualización, presupone que se trata de un instinto innato del organismo para desarrollar o conservar sus potencialidades.

Hasta aquí, lo escrito solo son algunas de las cosas que he obtenido, sin embargo, el mayor reconocimiento que le doy a la equinoterapia es el que a diferencia de otras no trabaja solo con mi cuerpo como si solo fuese un objeto que necesita moverse.

El sentido de esta frase -de acuerdo con lo expuesto por Rogers (1988)- evidencia una profunda reflexión sobre la percepción del Yo mismo del paciente, la cual se construye en la medida en que el cliente se descubre durante el proceso de forma más positiva y congruente con respecto a sus posibilidades de mejora. En otras palabras, el paciente resignifica la percepción que tenía de sí mismo y logra el reconocimiento integral de su ser persona, en el cual la condición de discapacidad es únicamente parte de su mismo ser.

La equinoterapia me permite recuperar sensibilidad corporal y experimentar emociones diferentes: felicidad, logros, orgullo, cariño, bienestar, entre otras emociones que, sinceramente, son necesarias para seguir adelante no solo con la terapia, sino también con la vida.

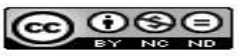

Esta obra está bajo licencia internacional Creative commons Reconocimiento-Nocomercial- 
Lo anterior constituye uno de los pasajes más emocionantes del discurso porque revela un sentido de cómo vivir, del poder de la elección, de la fe que se eleva por encima de la desesperanza y del vivir responsable con el que se enfrenta la cotidianidad.

De acuerdo con Rogers respecto a la utilidad del diagnóstico en la planeación de la terapia, se debe entender que más allá de la biología el verdadero problema de la discapacidad se halla, en última instancia, en una resultante cultural que niega el acceso de las personas a la vida plena, con lo cual se obstaculizan sus posibilidades de adaptación al entorno social.

En tal sentido, se debe destacar que el beneficio inmediato que proporciona la relación humano-equino ofrece la oportunidad de establecer contacto con un animal con el que, en cierto modo, podemos identificarnos en virtud de que sus reacciones constituyen un reflejo de nuestras propias actitudes de aproximación o de rechazo hacia la interacción con nuestros pares.

Rogers (1992) menciona que para muchos pacientes la relación con el terapeuta puede ser, quizá, la primera experiencia de aceptación incondicional que recibe, lo cual es justamente lo que el paciente percibe en su relación con el caballo; es decir, el caballo no evalúa la condición del jinete ni establece juicios de ningún tipo: solo acepta el contacto con la persona y accede a la colaboración solicitada. Sin embargo, el caballo puede, en ocasiones, experimentar una cierta indisposición o reticencia durante la monta, lo cual comunica con gestos posturales y movimientos impacientes, como mostrando desacuerdo con las actitudes del jinete, quien, en definitiva, debe restablecer la condición de confianza y respeto para reanudar el acuerdo de colaboración.

Otros autores como Chardonnens (2009) argumentan (teniendo a Rogers como referencia) que el caballo presenta como coterapeuta, en virtud de sus características y reacciones, importantes similitudes con las condiciones del terapeuta para la facilitación del proceso de cambio. Brühwier (citado por Chardonnens, 2009) señala que la finalidad de la terapias asistidas con equinos tiene como base del proceso de recuperación la lectura constante del lenguaje corporal del binomio equino-humano, lo que da lugar a la formación de un lazo de empatía recíproca durante la monta que, por cierto, se mantiene de manera perdurable.

\section{(c) $\rightarrow \& \Theta$}




\section{Revista Iberoamericana de las Ciencias de la Salud}

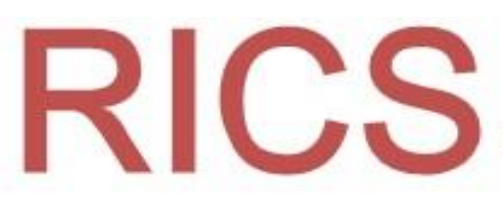

En este mismo sentido, Chardonnes (2009) apunta el planteamiento de Behr (otro importante autor con enfoque rogeriano), quien menciona dos elementos que siendo fundamentales para el éxito terapéutico del ECP, son extrapolados en la TEA: la empatía y la autenticidad de los terapeutas con sus clientes. A esta idea se debe agregar que las condiciones de empatía y autenticidad no se limitan al terapeuta y a su cliente, sino que resultan incluyentes y permean la tríada terapéutica (cliente, caballo y terapeuta) durante prácticamente todo el proceso.

Evidentemente, la función de acompañamiento y la propia resonancia afectiva del terapeuta son actores que permiten al paciente verbalizar su experiencia corporal sensorial, sus percepciones psicológicas, sus emociones y sus necesidades. Además, serán las cualidades del caballo las que faciliten el inshigth del paciente.

En síntesis - $-\mathrm{y}$ recapitulando las observaciones realizadas a lo largo de este documento, y para concretar en una frase la utilidad del enfoque centrado en la persona como fuente o recurso de análisis del proceso de la equinoterapia, aunque en este caso de las terapias asistidas con equinos en general - cabe señalar lo siguiente: durante el proceso de la interacción hombre-equino —en el contexto de una relación terapéutica- ocurre una condición asociativa (de contacto) que favorece la creación de un vínculo afectivo (aprecio positivo incondicional) que da lugar a la formación de una condición de confianza y apertura a la experiencia (congruencia). De este modo se crea un puente de comprensión recíproca (empatía) observable como situación de comunicación interespecies (equus-diálogo) que sirve para potenciar los efectos físico-psicológico-sociales y espirituales del cliente o paciente.

Por último, se debe considerar que dado el carácter tácitamente pre paradigmático del estado de las terapias asistidas con equinos, la asociación entre los fundamentos teóricos y los momentos del proceso que han sido expuestos constituyen per se una articulación temática cuyo carácter transdisciplinario propicia la transferencia de los saberes requeridos para dotar de una teorética propia a esta nueva disciplina.

\section{(c) क्S 9}

Esta obra está bajo licencia internacional Creative commons Reconocimiento-Nocomercial- 
Al hombre que duerme en nosotros: preguntarnos a cada instante si hombres somos y a cada instante contestarnos: no. El boceto somos mal pergeñado del hombre, aún por dibujar. Un pobre telón para la gran escena. ¿Hombres nos llamáis? Aún no: aguardad (Jura Soyfer, citado por Frankl, 1983).

\section{Referencias}

Artiles Monteagudo, M., Artiles Rivero, C. y Rodríguez Gómez, F. (2016). El estudio de casos como método problémico en ciencias médicas: una experiencia necesaria. Edumecentro, 8(1), 165-173. Recuperado de http://scielo.sld.cu/scielo.php?script=sci arttext\&pid=S207728742016000100013\&lng=es\&tlng=es.

Barceló, T. (2012). Carl R. Rogers y Eugene T. Gendlin: la relación que configuró un nuevo paradigma. Revista $\quad$ Focusing. Recuperado https://focusingelportalinterno.wordpress.com/2012/11/15/carl-r-rogers-y-eugene-tgendlin-la-relacion-que-configuro-un-nuevo-paradigma/.

Barreiro, M. S. (2015). La experiencia de pérdida de un ser querido. México: Instituto de Psicología y Educación. Universidad Veracruzana. Recuperado de https://cdigital.uv.mx/handle/123456789/46890.

Bautista, C. (2011). Proceso de la investigación cualitativa. Epistemología, metodología y aplicaciones. Colombia: Manual Moderno.

Bender, R. (2012). Hipoterapia. Chile: Mediterráneo.

Burbridge, V. (2004). El caballo en el arte: la prehistoria. Revista de Artes, (4). Recuperado de www.revistadeartes.com.ar/revistadeartes\%204/caballo.htm.

Castillo, M. C. (2011). Equinoterapia. Historia. Centro de Equinoterapia. Fundación Santa $\begin{array}{llll}\text { María Polo. Recuperado de } & \text { P }\end{array}$ http://actividades.uca.es/compromisoambiental/2011/C02/3.

Caudet, F. (2002). El caballo: más que un amigo. España: Editores Barcelona.

Chardonnens, E. (2009). The use of animals as co-therapist on a farm: The child horse bond in person centered equine-assisted psychotherapy. Person-Centered and Experiential

\section{(c) 9 क्ष}


Psychotherapies, $8(4)$,

319-332.

Retrieved

from https://psycnet.apa.org/doi/10.1080/14779757.2009.9688496.

Ernst, M. y De la Fuente, M. (2007). Manual básico de hipoterapia. Terapia asistida con caballos. España: La Liebre de Marzo.

Frankl, V. E. (2009). El hombre doliente. Fundamentos antropológicos de la psicoterapia. España: Herder.

Gross, E. (2006). La rehabilitación por medio del caballo. México. Trillas.

Gutiérrez, G., Granados, D. y Piar, N. (2007). Interacciones humano-animal: características e implicaciones para el bienestar de los humanos. Revista Colombiana de Psicología, (16), 163-183.

Hernández, R., Fernández, C. y Baptista, M. (2000). Metodología de la investigación. México: McGraw-Hill.

Längle, A. (2008). Vivir con sentido. Aplicación práctica de la logoterapia. Argentina: Lumen.

Netscher, T. y Samaniego, R. (2012). Revelaciones equinas. México: Edición de Autor, S. A.

Pérez, L., Rodríguez, J. y Rodríguez, N. (2008). La equinoterapia en el tratamiento de la discapacidad infantil. Revista Archivo Médico de Camagüey, 12(1). Recuperado de http://scielo.sld.cu/scielo.php?script=sci arttext\&pid=S1025-

$\underline{02552008000100016 \& \operatorname{lng}=\text { es\&tlng=es. }}$

Roberts, M. (2006). La unión. El hombre que habla a los caballos habla a las personas. España: Ediciones Tutor S. A.

Rogers, C. R. (1992). Psicoterapia centrada en el cliente. México: Editorial Paidós.

Rogers, C. R. (1993). El proceso de convertirse en persona. México: Editorial Paidós.

Rogers, C. R. (1998). Terapia, personalidad y relaciones interpersonales. Buenos Aires (Argentina): Editorial Nueva Visión.

Sarmiento, S. (2003). Promoción del desarrollo humano: diseño de una experiencia educativa en la enseñanza de la psicología (tesis de maestría). Instituto de Psicología y Educación. Universidad Veracruzana. Xalapa, Ver. Recuperado de https://www.uv.mx/veracruz/uvca285

\section{(c) $\rightarrow \& \Theta$}

Esta obra está bajo licencia internacional Creative commons Reconocimiento-NoComercial- 


\begin{tabular}{|c|c|}
\hline Rol de Contribución & Autor (es) \\
\hline Conceptualización & $\begin{array}{l}\text { Salvador Ernesto Sarmiento Vega.- 30\% María Esther Barradas Alarcón.- 25\% } \\
\text { Javier López González.- 15\% Martha Lilia León Noris.- 15\% Mayra Helen Posadas } \\
\text { Tello.- 15\% }\end{array}$ \\
\hline Metodología & Salvador Ernesto Sarmiento Vega.- 50\% María Esther Barradas Alarcón.- 50\% \\
\hline Software & NO APLICA \\
\hline Validación & NO APLICA \\
\hline Análisis Formal & $\begin{array}{l}\text { Análisis cualitativo.- Salvador Ernesto Sarmiento Vega.- 50\% María Esther } \\
\text { Barradas Alarcón. - } 50 \%\end{array}$ \\
\hline Investigación & Salvador Ernesto Sarmiento Vega 90\% María Esther Barradas Alarcón 10\% \\
\hline Recursos & Salvador Ernesto Sarmiento Vega $100 \%$ \\
\hline Curación de datos & $\begin{array}{l}\text { Salvador Ernesto Sarmiento Vega.- 30\% María Esther Barradas Alarcón.- } 30 \\
\text { Javier López González.- 10\% Martha Lilia León Noris.- 20\% } \\
\text { Mayra Helen Posadas Tello.- 10\% }\end{array}$ \\
\hline $\begin{array}{l}\text { Escritura - Preparación del borrador } \\
\text { original }\end{array}$ & $\begin{array}{l}\text { Salvador Ernesto Sarmiento Vega.- 60\% María Esther Barradas Alarcón.- 25\% } \\
\text { Martha Lilia León Noris.- 15\% }\end{array}$ \\
\hline Escritura - Revisión y edición & $\begin{array}{l}\text { Salvador Ernesto Sarmiento Vega.- 30\% María Esther Barradas Alarcón.- } 30 \\
\text { Javier López González.- 10\% Martha Lilia León Noris.- 20\% } \\
\text { Mayra Helen Posadas Tello.- 10\% }\end{array}$ \\
\hline Visualización & Salvador Ernesto Sarmiento Vega.- 55\% María Esther Barradas Alarcón.- 45\% \\
\hline Supervisión & Salvador Ernesto Sarmiento Vega.- 55\% María Esther Barradas Alarcón.- 45\% \\
\hline Administración de Proyectos & Salvador Ernesto Sarmiento Vega.- 55\% María Esther Barradas Alarcón.- 45\% \\
\hline Adquisición de fondos & $\begin{array}{l}\text { Salvador Ernesto Sarmiento Vega.- 20\% María Esther Barradas Alarcón.- } 20 \\
\text { Javier López González.- 20\% Martha Lilia León Noris.- 20\% Mayra Helen Posadas } \\
\text { Tello.- } 20 \%\end{array}$ \\
\hline
\end{tabular}

